\title{
Correspondence
}

\section{Declining adherence is a more likely explanation than frailty of the apparent decline in efficacy in the CAPRISA 004 trial}

(AIDS 2012, 26:17.p 2261.)

Grobler, Anneke ${ }^{\mathrm{a}}$; Abdool Karim, Salim ${ }^{\mathrm{a}, \mathrm{b}}$

Author Information:

${ }^{\mathrm{a}}$ Centre for the AIDS Programme of Research in South Africa (CAPRISA), University of KwaZulu Natal, Durban, South Africa

${ }^{\mathrm{b}}$ Department of Epidemiology, Mailman School of Public Health, Columbia University, New York, New York, USA.

Correspondence to Anneke Grobler, CAPRISA, University of KwaZulu-Natal, Durban, South Africa. E-mail: grobler@ukzn.ac.za

O'Hagan et al. [1] offer an alternate explanation for the apparent decline in efficacy over time in vaccine and microbicide trials. They propose that the decline in efficacy observed could also be explained by selection bias due to heterogeneity in infection risk. As those at highest risk are infected more in the placebo arm than in the active arm, it leads to differences in the composition of the study population in the two study arms, a phenomenon called frailty in statistics [1].

There are several potential reasons for the apparent decline in efficacy in trials. However, identifying one or more reasons within a trial is a challenge. Using the CAPRISA 004 microbicide trial [2] as an example, we examined three possible reasons for the observed declining efficacy over time.

First, the decline in efficacy over time could, as suggested by O'Hagan et al.[1], be due to frailty. Analysis of the trial data, however, did not reveal evidence of variation in the timespecific rate ratios. When the interaction between treatment arm and time (on a logarithmic scale) was included in a proportional hazards model, the coefficient of the interaction term was $0.31(95 \%$ confidence interval -0.19 to $0.82 ; \mathrm{P}=0.23)$. Given this test has limited power, failure to reject the null hypothesis cannot be taken as evidence that there was no attenuation over time.

Although, theoretically, frailty is a plausible explanation, it is unlikely to be the predominant reason for the decline in efficacy over time in the CAPRISA 004 trial. Frailty is likely to have a large impact when the incidence of disease is high [1] and/or the incidence rate difference between the study arms is substantial. In the CAPRISA 004 trial, there were only 22 additional infections in the placebo group, resulting in only 22 women in a group of more than 400 women, who contributed to the changing risk set in the placebo arm. This number is unlikely to be large enough to make a substantial difference. Hence, frailty is unlikely to be the primary driver of declining efficacy in the CAPRISA 004 study. 
A second possible explanation for declining efficacy over time could be biological, for example, increasing tenofovir resistance over time could lead to reduced efficacy. However, no evidence of resistance to tenofovir was found in the CAPRISA 004 study, making this an unlikely explanation for the decline in efficacy over time.

Finally, adherence to correct and consistent use of a microbicide might wane over time, leading to lower efficacy. Adherence in the CAPRISA 004 study was assessed by participant self-report and by counting the number of used applicators returned each month. The median number of returned empty applicators per month was shown to decline over the first 18 months on study. The median number of returned used applicators per month was eight in the first 6 months of the study, six from 6 to 12 months and four from 12 to 18 months on the study. Preliminary analysis of vaginal tenofovir concentrations demonstrates a similar decline in detectable tenofovir over time. This offers the most plausible reason for the observed temporal declines in efficacy.

In conclusion, we thank the authors for highlighting frailty as a potential explanation of declining efficacy. In the CAPRISA 004 trial, where an apparent decline in efficacy is seen, we do not find empiric evidence of frailty, but we do see evidence of declining adherence over time.

Acknowledgements

Conflicts of interest

There are no conflicts of interest.

References

1. O’Hagan JJ, Hernan MA, Walensky RP, Lipsitch M. Apparent declining efficacy in randomized trials: examples of the Thai RV144 HIV vaccine and South African CAPRISA 004 microbicide trials. AIDS 2012; 26:123-126. doi: 110.1097/QAD.1090b1013e32834e32831ce32837.

2. Abdool Karim Q, Abdool Karim SS, Frohlich JA, Grobler AC, Baxter C, Mansoor LE, et al. Effectiveness and safety of tenofovir gel, an antiretroviral microbicide, for the prevention of HIV infection in women. Science 2010; 329:1168-1174. 